\title{
Considerations for Web of Object Service Architecture on IoT Environment
}

\author{
NamKyung Lee*, HyunWoo Lee* and Won Ryu* \\ * Electronics and Telecommunications Research Institute \\ nklee@etri.re.kr
}

\begin{abstract}
The internet is an optimal place to human as a producer or a consumer for sharing information until now. In the future, not only the human-generated information but also things will be connected into internet and these will evolve into internet of things(IoT) which can share the information thing-in-itself and its environment. Firstly, this paper proposes a service architecture which includes various devices for providing web base IoT services. Secondly, this paper also proposes a service platform which supports service orchestration and composition with device objectification and finally describes structure and functionalities of web of object gateway.
\end{abstract}

Keywords: IoT, Service Architecture, Web of Object, Device Objectification

\section{Introduction}

Current internet is evolving into internet of things(IoT) which can connect with various information-thing itself and information environment.

The internet of thing (IoT) environment means that various devices which compose IoT provide services with connection and communication each other without user's recognition or interference. Current M2M systems are designed for providing a service in special domain. For such reasons, the system occurs high development and management cost for installation, extension and maintenance. It causes increasing demand of common M2M platform works with various domains.

The web of object in IoT objectifies and virtualizes things. And it generates and provides new services with combination of objectified things with context information. The thing in the web of object is embedded with web protocol and provides web services with things. But, most of things today does not use HTTP protocol and uses light-weight protocols as like Zigbee, Bluetooth, etc. For inclusion of current things to IoT environment, it requires IoT service mapping between the thing and web service environment and extension of service collaborations on current wire/wireless home network [2].

This paper concerns IoT service environment with device objectification and attempts to provide user-oriented device web service with service overlay network.

\section{Related Works}

\subsection{DIYSE Project}

DIYSE(Do It Yourself Smart Experiences) project[3] seeks personalization and interaction with each other on IoT environment as like DIY. It makes household goods have been recognized in network and connected to each other things. User can create and combine a new service with the connected things. The DIYSE is a basic step of IoT service and requires intelligent device to apply devices to pre-defined services. It has limitation to create new services with various things. 


\subsection{DPWS}

DPWS(Device Profile for Web Services)[4] specifies device profiles for providing web service in embedded devices. The DPWS protocol provides event functionalities (messaging, service discovery, security, profile, resource, etc.) for device. DPWS has two types of services. One is hosting service and the other is hosted service. The devices will notify their information, connect and control each other in hosting service. The hosted service will be provided related functionalities by hosting service.

\subsection{SOCRADES Project}

SOCRADES project[5] proposes SIA(SOCRADES Integration Architecture) which provides various mash-up services with connected devices. The SIA also provides realtime monitoring and management for devices and exchanges profile and information with DPWS or REST supported devices. It includes modeling of hardware and software, system modeling, design, functionality, etc.

\section{Web of Object Service Architecture}

A web base web of object service requires interoperability, generality, communication efficiency, mobility, intelligence, etc. And it also requires management functionalities for service, location, context, error, traffic, security, privacy, etc.[6]

\subsection{Smart home Web of Object Architecture}

This paper proposes a web of object service architecture for smart home service environment. As the smart web of object architecture (called SWOA) uses web service platform structure, it can be applied to multiple users and current service environment easily.

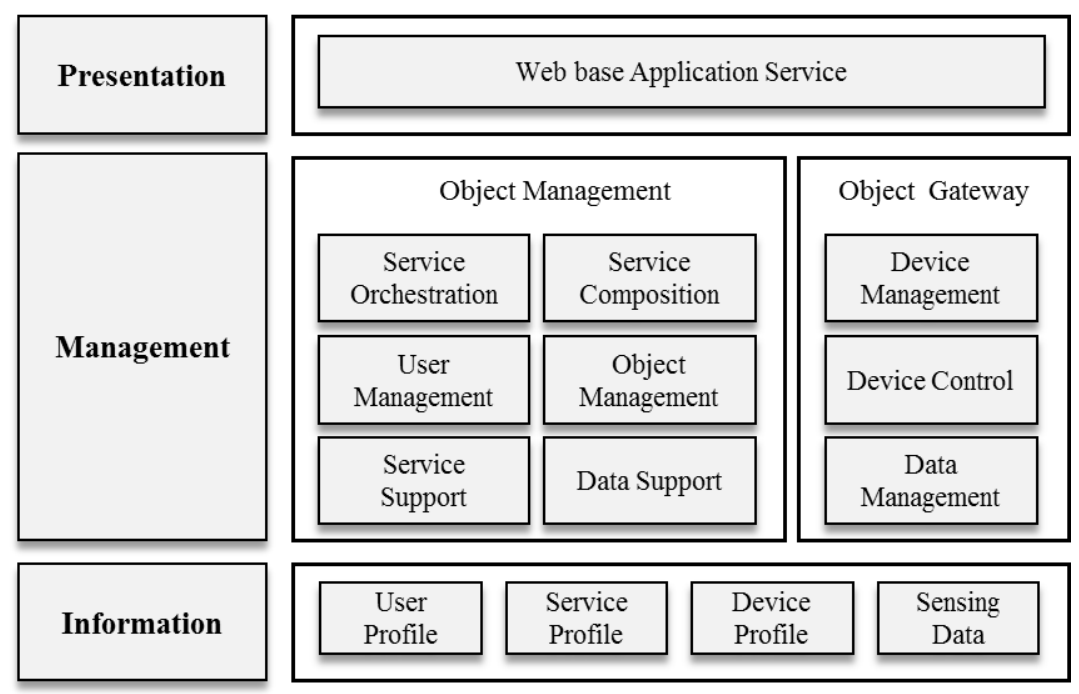

Figure 1. Smart home Web of Object Architecture

Figure 1. shows SWOA and it consists of presentation layer, management layer and information layer. The presentation layer is an application which provides web base service to users and the management layer works for object management and object gateway. The object management includes management of user, service and distributed sensor gateway. It performs service orchestration which works for inter service domains and composition which works for intra service domain. The sensor gateway manages 
devices, device controls and device sensing informations. The information layer gathers user, service, device and sensing data informations.

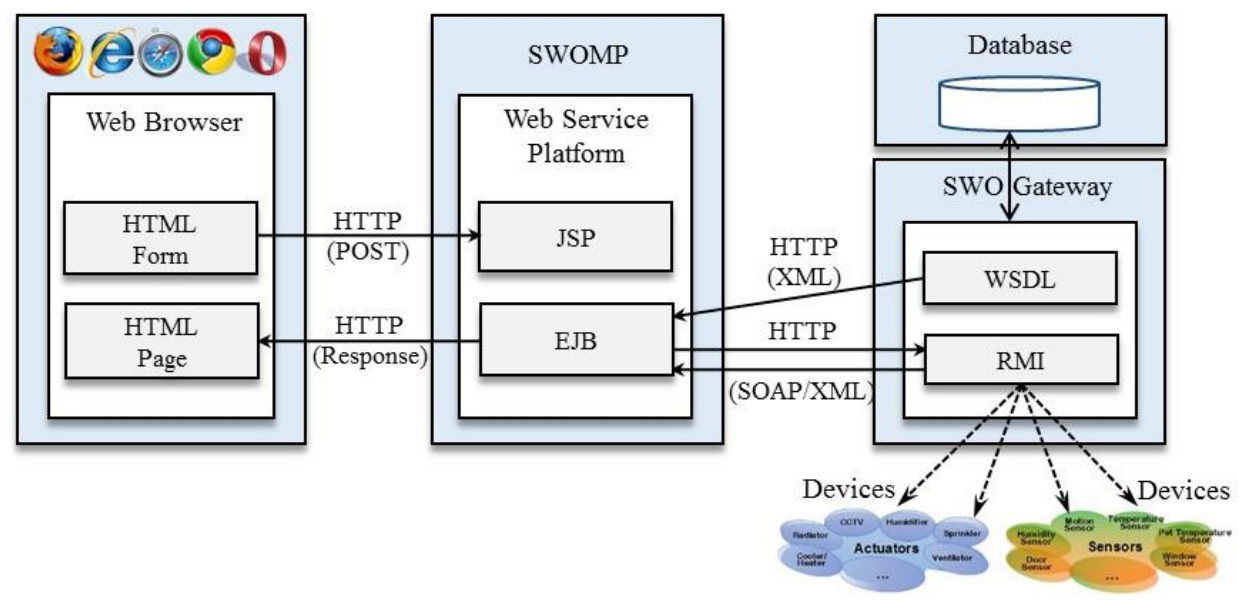

Figure 2. Interface Environment of SWOA

Figure 2 shows interface environment between each of the systems in SWOA. A user application is provided as web service via web browser and the user can use services easily with web page form and etc.

The SWOA provides device web services with smart home web of object gateway (SWO Gateway, SWO GW) which manages devices and provides web connectivity to Non-IP devices. This paper adopts service overlay network concept for providing new service by service orchestration and service composition. The smart home web of object management platform (SWOMP) which is located in management layer of SWOA provides various device web services as like composition, service management, context management, error handling, etc. with user, service, device profile and sensing information from SWO GW.

\subsection{Smart home Web of Object Management Platform}

Figure 3 shows functional view of SWO management platform consisted of service orchestration, service omposition, objects, sensor data support, and service support functions. Sensors and actuators are divided into web-supported and not web-supported objects and sensor gateway is used for supporting not web-supported objects. The service composition consists of follwoing basic functions as illustrated in the figure 3 .

- Registry : Registry function describes and publish the offered functionality of the services to potential consumers (clients). A service registry allows you to organize information about services and provide facilities to publish and discover services.

- Discovery \& Selection: Service discovery \& selection function is the process of locating and gaining access to provided services that satisfy a set of requirements (whether it is capabilities or non-functional aspects).

- Deployment : Service deployment function involves concretely associating services to devices in the real world system that is used as the infrastructure

- Exposure : Depending on whether the service provider or owner decides to exposes such information or not.

- Security: Providers have to guarantee the delivered service to respect a given security policy, in any interaction with the operational environment, and regardless who actually called the service. 
- Session Control: Session control function deals with the network call control interactions.

- Service Management : Service management function comprises all the mechanisms that are required for capturing and processing several attributes of a service execution (related but not limited to quality aspects) to determine whether predefined agreements (such as Service-Level Agreements) are abided by and the execution goes as planned.

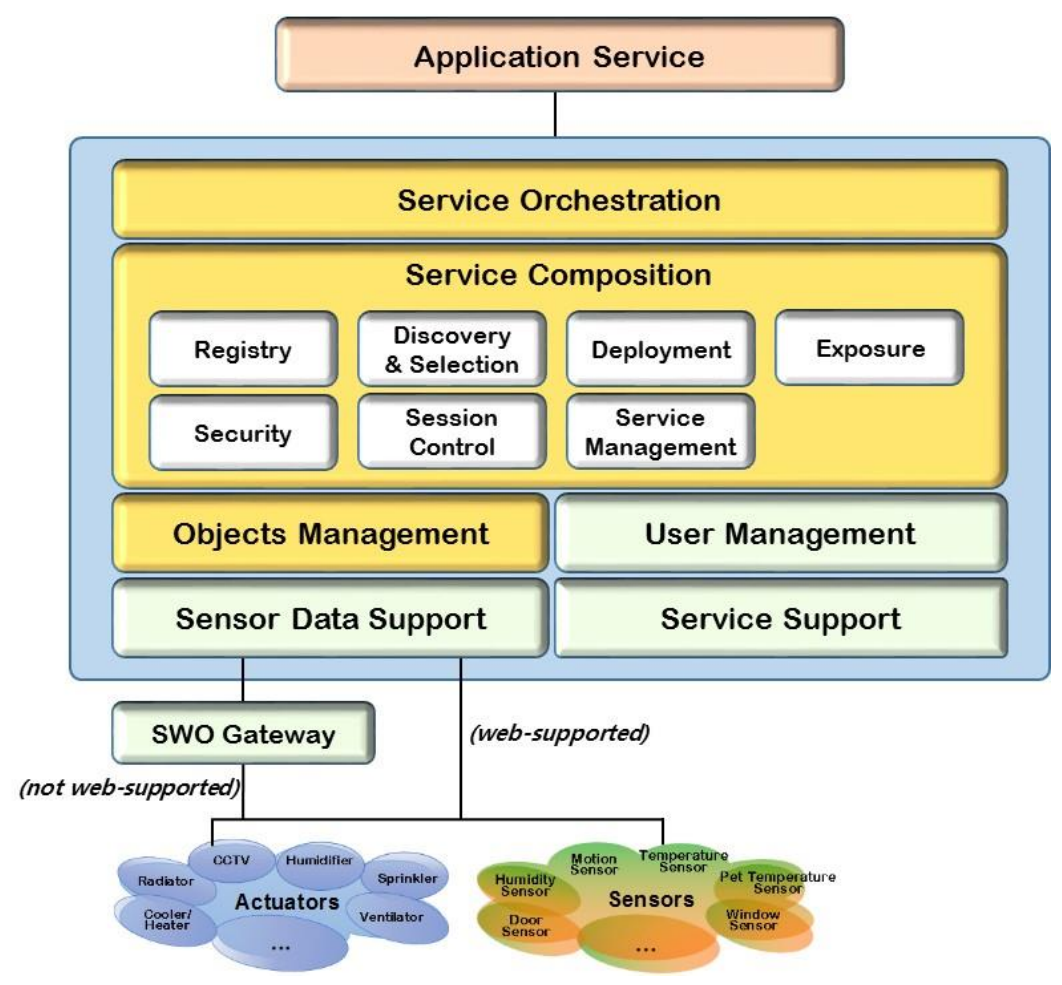

Figure 3. Smart Web of Object Management Platform (SWOMP)

\subsection{Smart Web of Object Service Model}

Users and devices in physical world will be service resources by objectification and the object means that it exists on web.

Web base smart web object service environment is not just provides service by translate a device into an object. Because it provides new IoT service by combination of various objects and components with objectified devices, this paper adopts service overlay network concept.

SWOMP considers four planes (user, service, object and physical plane). The device objectification and concept of service overlay workflow in SWOMP is shown in figure 4.

- User plane provides services. It delivers service request and context information to the object plane. Also, it receives service allocation information from the object plane.

- Service plane combines and coordinates a set of services as mash-ups. In figure 4, it has four- pet care, auto ventilation, plant water supply, and home security services.

- Object plane objectifies physical devices as web of objects with concept of device virtualization. It manages sensor object, actuator object, and service enablers. It also delivers service response to the user plane. 
- Physical plane accesses and controls physical devices such as CCTV, humidifier, sprinkler, ventilator, cooler, heater, and radiator. Also, they manage actuators like motion, temperature, pet temperature, window, door, and humidity sensor.

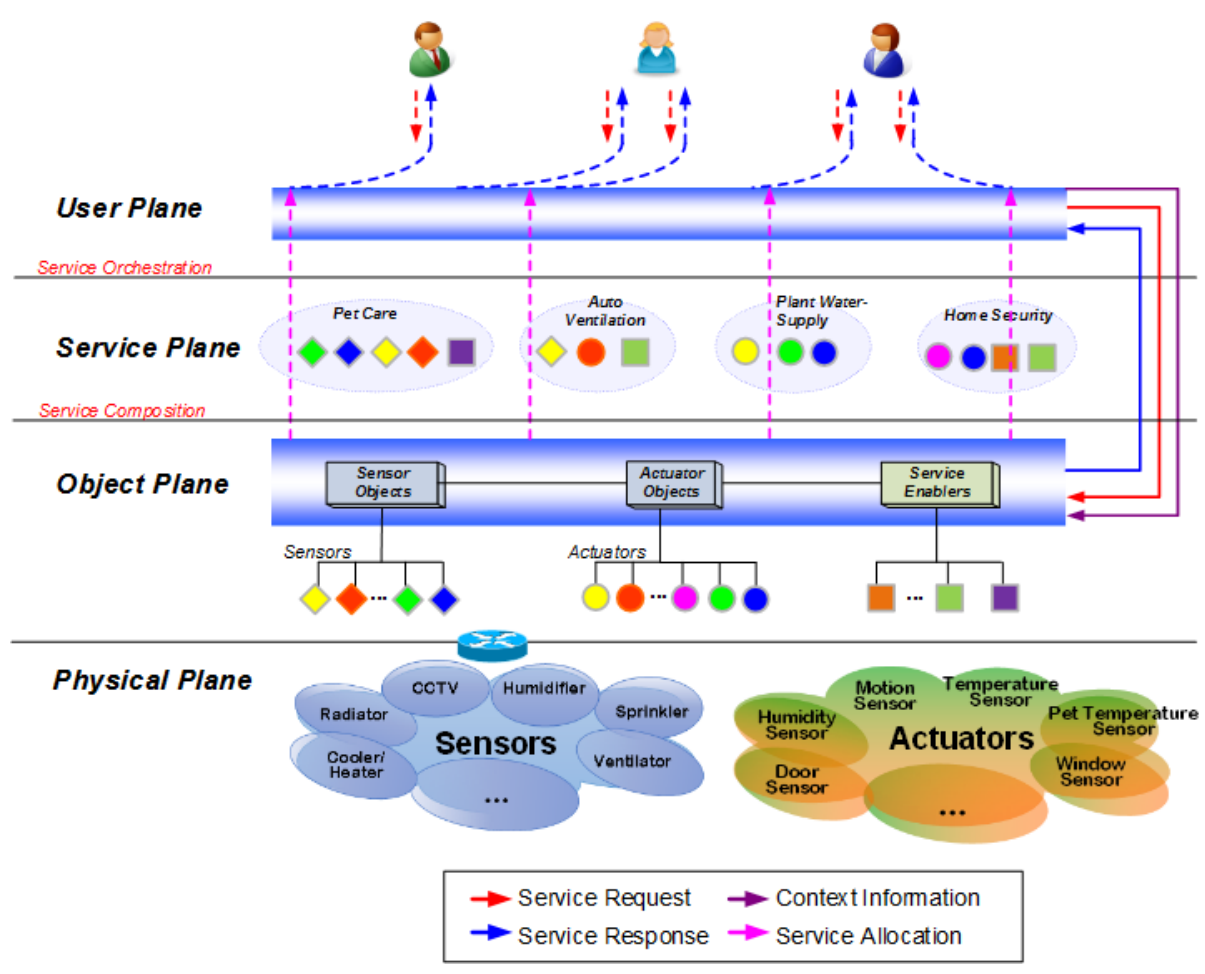

Figure 4. Service Flow of Web of Object Service Platform

\section{Smart home Web of Object Gateway and Device Objectification}

\subsection{Smart Web of Object Gateway (SWOGW)}

SWOGW performs device registration, device profile management, sensing information management, device control, device web service scheduling, etc. The SWOGW receives service request messages and generates response messages.

Figure 5. shows the functional structure of SWOGW with various device web services processing functions. The SWOGW locates between SWOMP and device. It works with database for storing and management of devices information in its domain. 


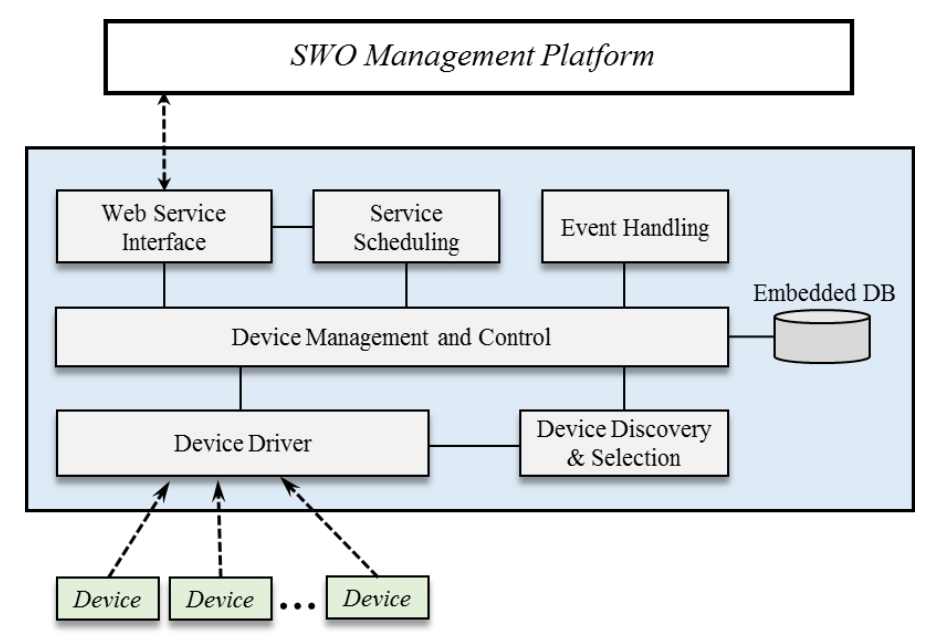

Figure 5. Functional Structure of SWOGW

\subsection{Device Objectification}

Device objectification makes a non-IP thing or a device to be an object which enables service collaboration for providing device web service by SWOGW.

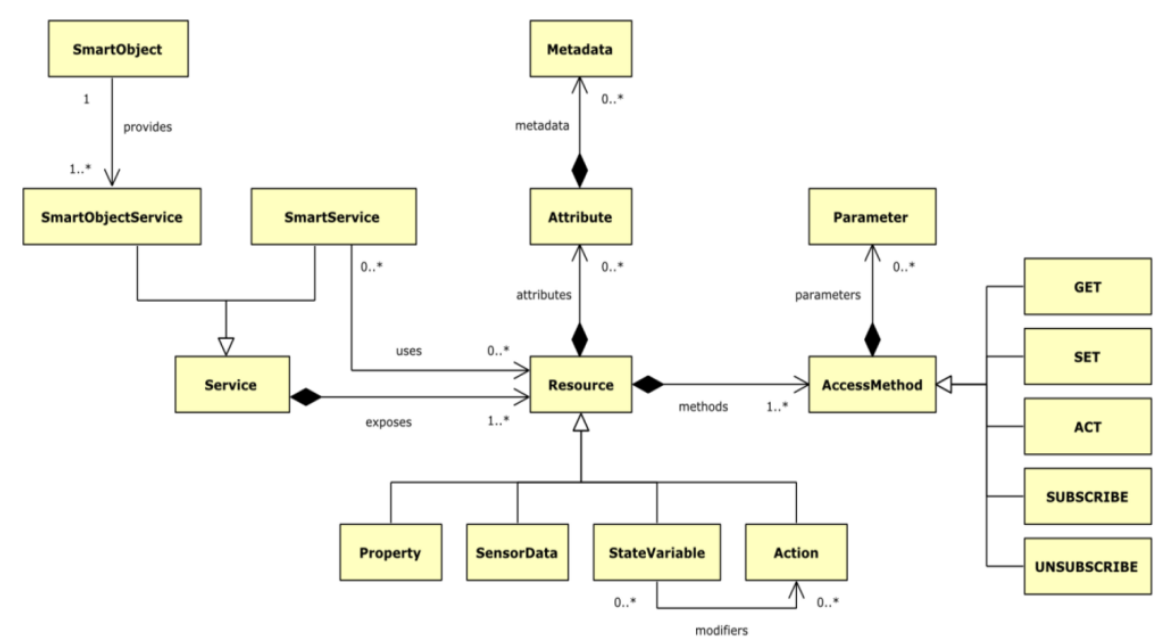

Figure 6. Relation of Smart Home Object and Service

This paper modifies DPWS(Device Profile for Web Services) base device profile for smart web of object services for device objectification. By means of device objectification, all of devices can have their own profile, method and control information.

For providing web services on IoT environment, device profile can be achieved by providing WSDL (Web Service Description Language) based on SOAP. 


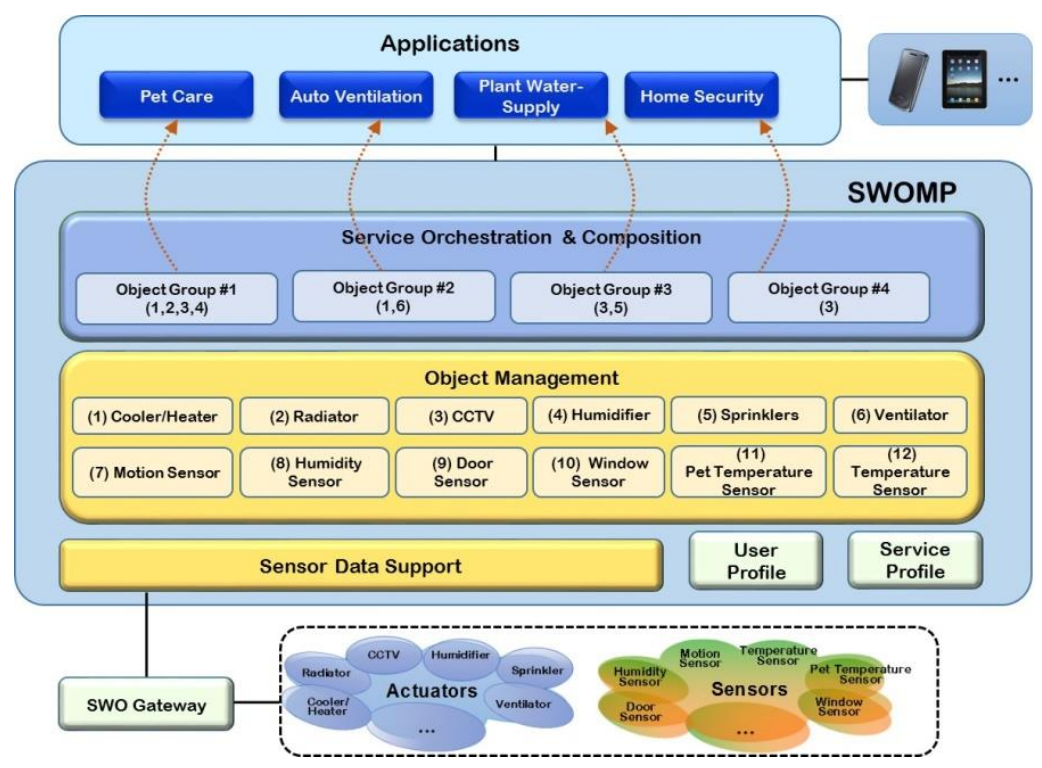

Figure. 7. Device Objectification and Service Deployment over SWOMP

\section{Conclusion}

The device objectification which extends current IoT research and enables various IoT services is very important issue. Device objectification makes a non-IP thing or a device to be an object which enables service collaboration for providing device web service by SWOGW.

This paper defines smart web of object service architecture (SWOA) for providing IoT service and describes structure and functionalities smart web of object platform (SWOMP), gateway (SWOGW) and device orchestration.

For further study, it is needed that research about lightweight protocol and service orchestration with value-added service by 3 rd party service providers.

\section{References}

[1] Y. J. Kim, Y. K. Jeon, and I. Y. Chong, "Intelligent IoT Service with Device objectification mechanism", Proc. KICS Conf., (2013), p.1.

[2] D.C.Kim and K.S.Chung, "Design of system architecture for device collaboration service in home network environments", in Proc. KICS Conf., (2011), p. 1.

[3] http://www.dyse.org

[4] http://docs.oasis-open.org/ws-dd/ns/dpws/2009/01

[5] http://www.socrades.eu

[6] Y. J. Kim, Y. K. Jeon, and I. Y. Chong, "Service overlay network platform and service composition method for M2M service" in Proc. Korea Computer Congress, (2012).

\section{Authors}

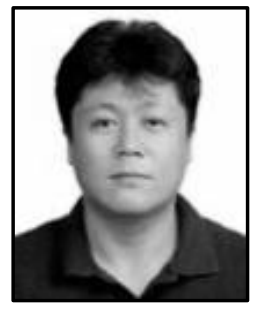

NamKyung Lee, he received BS, MS, Ph.D. degrees in computer engineering from Korean Aviation University, Korea, in 1996,1998 and 2005 respectively. He joined ETRI in 2000 and has been involved with IMSCB, MOBISAT development with satellite communication and open IPTV platform development. He led web application virtualization system development with KT and web of object service platform with ITEA2. He is currently working as an editor of IEEE P1903 and senior engineer of media networking research team. 


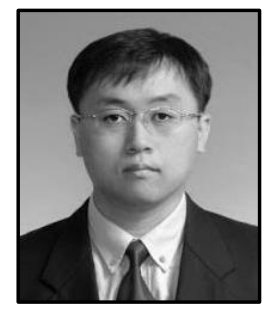

HyunWoo Lee, he received the B.S., M.S. and the Ph.D. degree in the School of Electronics, Telecommunication and Computer Engineering at Korea Aerospace University, Korea in 1993, 1995 and 2005 respectively. From 1995, he has joined Electronics and Telecommunications Research Institute (ETRI). From 2009, he is a team leader in the Smart Screen Convergence Research Department in ETRI. His research interests include IPTV service, service control and traffic congestion control.

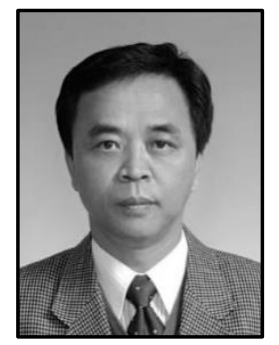

Won Ryu, he was born in Busan, South Korea, in 1958. He received the B.S. degree in the department of computer science \& statistics from Pusan National University, Busan, South Korea, in 1983, and the M.S. degree in the department of computer science \& statistics from Seoul National University, Seoul, South Korea, in 1988, respectively, and his Ph.D. degree in information engineering from SungKyunKwan University, Kyonggi, South Korea, in 2002. Since 1989, he has been joining as a researcher and currently he is a project leader in the Broadband convergence Network Interworking Research Laboratory, Electronics and Telecommunications Research Institute (ETRI), Daejeon, Korea. His research interests include All-IP based Convergence Service \& Control Plane, Mobility, NGN, Wire/Wireless Interworking Technology, and VoIP. 